\title{
Long-term outcome after laparoscopic and open surgery for rectal prolapse
}

\section{A case-control study}

\author{
Y. Kariv, ${ }^{1}$ C. P. Delaney, ${ }^{2}$ S. Casillas, ${ }^{1}$ J. Hammel, ${ }^{3}$ J. Nocero, ${ }^{1}$ J. Bast, ${ }^{1}$ K. Brady, ${ }^{1}$ V. W. Fazio, ${ }^{1}$ A. J. Senagore ${ }^{4}$ \\ ${ }^{1}$ Department of Colorectal Surgery, Cleveland Clinic Foundation, Cleveland, OH, USA \\ ${ }^{2}$ Division of Colorectal Surgery, University Hospitals of Cleveland, 11100 Euclid Avenue, Cleveland, OH 44106, USA \\ ${ }^{3}$ Department of Quantitative Health Sciences, Cleveland Clinic Foundation, Cleveland, OH, USA \\ ${ }^{4}$ Department of Surgery, Medical College of Ohio, Toledo, OH, USA
}

[Surg Endosc (2006) 20: 35-42, DOI: 10.1007/s00464-005-0096-7]

This article has been published online as DOI: 10.1007/s00464-005-3012-2. The publisher apologizes for any confusion. 